\title{
A research team's perspective on the editorial "Revolutionary reform in psychiatric care in Italy: The abolition of forensic mental hospitals"
}

\author{
Claudio Di Lorito, Tom Dening \& Birgit Völlm \\ Division of Psychiatry and Applied Psychology, School of Medicine, \\ University of Nottingham, United Kingdom
}

The editorial by Traverso \& Traverso "Revolutionary reform in psychiatric care in Italy: The abolition of forensic mental hospitals" represents key work to raise awareness around the reform which led to the definite closure of forensic psychiatric hospitals in Italy. Given the revolutionary underpinnings and implications of Law 81/2014 and that little is known about the reform, such work is crucial, as it encourages reflection among professionals, clinicians, academics and policy makers. Cost optimisation, reducing length of stay and timely transition from secure to community-based services are shared priorities in different countries. We deem it equally essential to add empirical research to the discourse around the reform, which, as highlighted by Traverso and Traverso 'ensures the progress made is defensible'.

Inspired by the potential implications of the reform well beyond the Italian borders and immersed in an academic culture which values work at the interface between research and practice, our research team based at the UK University of Nottingham collaborated with a group of Italian psychiatrists to conduct some initial research around patients' experience of the Italian service. The research originated from a EU-funded Short Term Scientific Mission of the European Cooperation in Science and technology (COST) Action 'Towards an EU research framework on forensic psychiatric care'. The Action funded a member of our research team to conduct research in the Residential Units for the Execution of Security Measures (Residenze per l'Esecuzione delle Misure di Sicurezza - REMS) located in Castiglione Delle Stiviere, Lombardy. 
Given this work (Di Lorito et al., 2017a and b), we read the Traverso's editorial with great interest. The authors' recommendations about the REMS seemed particularly salient - that they should: (1) be 'constructed with a real therapeutic milieu'; (2) 'encourage cooperation [among patients] in all aspects of daily life'; and (iii) 'empower patients and enable them to engage with the positive values of the wider society'. We contribute some of our initial research findings, in the hope that they will further discourse in this area.

\section{1. 'A real therapeutic milieu'}

Emphasis on the therapeutic mission of REMS is evident in Law 81/2014, which defines the scope and regulations of the new service. In line with the ethos of the United Nations Convention on the Rights of Persons with Disabilities, Law 81/2014 mandates that a patient cannot stay in the service for a period longer that a prison sentence for the same index offence. This is crucial to encourage a therapeutic approach and promote timely discharge into community mental health services. Our research findings suggest that the principles outlined in policy documents have been translated in daily practices at the REMS Castiglione delle Stiviere. The participants appreciated, among other aspects related to care and treatment, the effort of members of staff to go beyond traditional healthcare duties and establish a truly human connection with them, as reflected in one patient's words: 'I see there is an interest in my emotional wellbeing. When I am sad, they invite me to talk'. The genuine commitment of the staff to the patients' emotional wellbeing was reported to enhance clinical rapport and the therapeutic journey of the participants.

\section{2. 'Cooperation [among patients] in all aspects of daily life'}

The humane dimension of the recovery philosophy was also evident in the socially-oriented atmosphere that we observed at the REMS Castiglione delle Stiviere. Having virtually unrestricted freedom of movement and unescorted access to all the facilities, the patients 
engaged spontaneously in organising social activities such as playing card games, discussing politics and chatting at the café. Most of our interviewees reported having a special friend or a small group of friends, with whom to spend quality-time and receive emotional and practical support. Cooperation and bonding represented essential coping mechanisms to deal with the emotional and practical difficulties of life in the REMS, as expressed by one patient: "I am happier on the days I know I will be going to the library and when I am with my friends and we play cards or bowling"

\section{3. 'Empower patients and enable them to engage with the positive values of the wider society'}

We gathered positive feedback around the opportunities offered to promote the patients' reintegration in the community. These include activities organised outside the institution, which ensure that the patients can socialise with "ordinary citizens", or temporary leaves, during which patients deemed fit upon clinical evaluation can spend private time outside the institution. Currently, around half of the patients at the REMS Castiglione delle Stiviere are granted at least one monthly leave of a few hours. The patients reported that these "allowances", by keeping them in touch with the outside world, boosted their commitment to the recovery process. Community-oriented activities were also found to promote sociallyintegrated lifestyle, as evidenced by the rare occurrence of serious incidents, despite (or because of) the absence of security/police forces. The clinical director of the REMS Castiglione delle Stiviere interpreted this successful result through a social-constructionist perspective, suggesting how in highly restrictive settings individuals may internalise a captive prisoner's self-identity and behave aggressively. By contrast, when patients are treated truly humanely, they tend to refrain from anti-social behaviour.

In conclusion, our findings evidenced some success in the long journey of reform of the forensic psychiatric sector in Italy in at least one of the specialist units. It is remarkable that 
patients who were once deemed to require high security are now accommodated in REMS which only employ clinical personnel and where the only security measures are a fenced perimeter, CCTV and airlocked doors. We understand that in some units, such as in the tiny four-bedded unit in Trieste, the unit remains completely unlocked throughout the day. This has had a positive impact on the ageing patients' overall service satisfaction and has not caused any discernible increase in adverse incidents. Our research also evidenced that further improvement of service provision is needed, including more consistent practical and emotional support to ensure that those patients who need motivation to remain engaged in the recovery process can develop that. Given the exploratory nature of our research, we were not able to provide any hard data on outcomes, so further systematic research, with more representative samples, is necessary, but these preliminary findings are promising.

The research was supported by the NIHR Collaboration for Leadership in Applied Health Research and Care East Midlands (CLAHRC EM). The views expressed are those of the author(s) and not necessarily those of the NHS, the NIHR or the Department of health.

\section{References}

Di Lorito, C. Castelletti, L., Lega, I., Gualco, B., Scarpa, F. \& Völlm, B. (2017a). The closing of forensic-psychiatric hospitals in Italy: determinants, current status and future perspectives. A review. Submitted for publication in the International Journal of Law and Psychiatry. Available from the first author.

Di Lorito, C. Castelletti, L., Tripi, G., Gandellini, M.G., Dening, T. \& Völlm, B. (2017b). The individual experience of ageing patients and the current service provision in the context of Italian forensic-psychiatry: A case-study. Submitted for publication in the Journal of Forensic Nursing. Available from the first author. 